\title{
Intestinal endometriosis
}

\author{
N. H. TOWNELL \\ F.R.C.S. \\ J. D. VANDERWALT \\ M.B., B.Ch.
}

Enfield District General Hospital (Chace Wing), Enfield, Middlesex

\section{Introduction}

Endometriosis is a strange and protean disease (Kistner, Siegler and Behman, 1977). Endometriotic deposits usually affect the genital organs and pelvic peritoneum but bowel, urinary tract and extraabdominal sites may also be involved (Ranney, 1975). Ranney (1975) believes the overall incidence of endometriosis to be between $8 \%$ and $30 \%$ of patients requiring gynaecological surgery. Macafee and Greer (1960) reviewed eight series of endometriosis in the world literature totalling 7,177 patients, and found intestinal involvement in $12 \%(880$ patients), affecting sigmoid or rectum in $72.4 \%$ of cases, rectovaginal septum $13.5 \%$, small bowel $7.0 \%$, caecum $3.6 \%$, appendix $3.0 \%$ and other sites in $0.5 \%$.

More recently Williams and Pratt (1977) set out to discover whether a predicted increase in endometriosis had occurred because of the gradual decrease in size of the average family. Recording a prospective series of 968 operations, they reported an overall incidence of endometriosis in 38\%. Intestinal involvement was present in $37 \%$ of those affected and urinary tract endometriosis was found in $16 \%$.

\section{Clinical presentation}

Extensive endometriosis of the ovaries may be virtually symptomless but four or five implants in tissues where room for expansion is limited such as the rectovaginal septum or scar tissue, produce excruciating pain. Kistner et al. (1977) listed the four cardinal symptoms of endometriosis as infertility, dysmenorrhoea with pelvic pain, dyspareunia and menstrual problems. Although endometriosis does not occur before the menarche and is usually confined to the reproductive years, it may present after the menopause due to the administration of exogenous hormones or due to progression of the scarring process. It is associated with primary infertility and may also develop during the deliberate long

Correspondence: N. H. Townell, F.R.C.S., Department of Urology, 7th Floor, The Royal Free Hospital, Pond Street, London NW3 2QG. period of infertility following early completion of a family. Endometriosis is uncommon within 5 years of 0 pregnancy. Dysmenorrhoea of endometriosis distin- 3 . guishes itself from primary dysmenorrhoea in that it 8 may commence at any time during the reproductive years and the symptoms are closely related to the first $\%$ day of the menstrual cycle, often peaking several $\rightarrow$ days before the onset of menstruation.

Intestinal endometriosis additionally produces 을 constipation and diarrhoea. These symptoms along with indigestion, nausea, vomiting, daily cramping and rectal pain may be unrelated to periods (Gray, 1973). The bowel mucosa overlying endometrial $\stackrel{\oplus}{\hookrightarrow}$ deposits is usually intact and rectal bleeding \& $\overrightarrow{0}$ subsequently unusual (Gray, 1973). The clinicial history may thus be very suggestive but periodicity ơ symptoms may not always be present and the patient can present with acute, partial or chronic intestinal obstruction (Ranney, 1975).

\section{Pathology}

The gastrointestinal symptoms may be explained by reviewing the pathological changes of intestinal endometriosis recently summarized by Morson and $\overline{0}$ Dawson (1979). On gross examination endometriomas (Fig. 1) are usually ill-defined indurated $\dot{0}$ tumours seldom larger than $5 \mathrm{~cm}$ in diameter, having a glistening grey colour on cross-section. Tiny areas $\delta$ of haemorrhage within the tumour are characteristic. $₹$ They are usually found in the sub-serous and $\frac{2}{9}$ muscular coats of the bowel wall (Fig. 2) and may $D$ project into the lumen as a polyloid mass covered by intact mucosa. Externally, they may closely mimic N carcinoma. Endometriosis presenting as multiple serosal deposits (Fig. 3) can be confused with O $^{\circ}$ secondary carcinoma or nodules of fat necrosis. Their haemorrhagic appearance should serve as a distingu- 0 ishing feature but histological examination may be 0 required. Microscopically, islands of endometrial glands and stroma are seen between muscle bundles $\stackrel{?}{?}$ or in the serosa. Inflammatory cells are often present $\square$ and macrophages laden with haemosiderin derived $\bar{O}$ 
from episodes of haemorrhage are frequently seen. The characteristic induration is mostly due to muscular hypertrophy, but fibrosis resulting from repeated minor haemorrhages in the tumour mass is also an important factor. It is this induration and fibrosis that produces most of the lesions likely to present to the general surgeon.

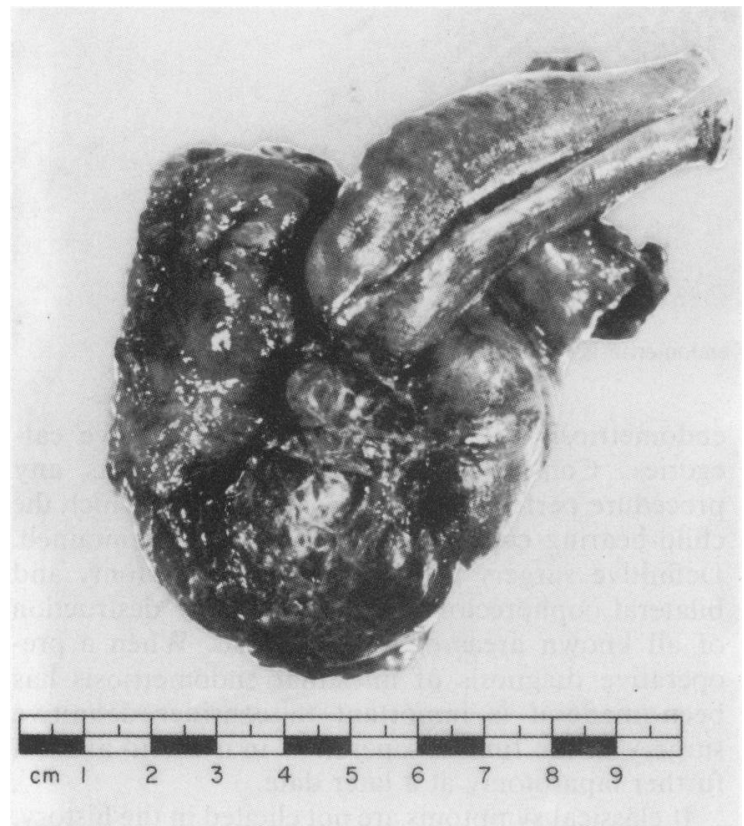

FIG. 1. Endometriosis arising in the caecum and involving appendix and terminal ileum. The ileum has been opened to the point of partial obstruction of the lumen.

\section{Physical examination}

The clinical history is undoubtedly the most important factor in making the diagnosis but physical examination may provide useful corrobarative evidence. The patient with intestinal endometriosis may only exhibit signs referable to that lesion, but other features of endometriosis are often present. Both rectal and vaginal examinations are thus essential, the most common findings being small, often tender, nodules behind the uterine cervix. There may also be an enlarged uterus and ovaries with some degree of fixation due to adhesions. If possible, it is advisable to perform a pelvic examination just prior to, or during menstruation, as signs are often maximal at this time (Kistner et al., 1977). Repeating the examination between menses may reveal a change in the degree of tenderness or size of any mass, this being useful confirmatory evidence of endometriosis (Ranney, 1980).

\section{Investigations}

Sigmoidoscopy is often difficult due to tenderness of the pelvic organs, but barium enema may be useful, although not always diagnostic (Burns, 1967). Classically, when compared to colonic carcinoma, large bowel endometriosis demonstrates a longer filling defect, intact mucosa and inconstancy in the size of the lesion (Burns, 1967). More recently, ultrasound has been used in an attempt to improve diagnostic accuracy. Sandler and Karo (1978) state that the finding of a cystic lesion with an irregular 'shaggy' wall and some evidence of septation should suggest endometriosis, but they also found that they could not distinguish it with certainty from similar ultrasonic appearances due to pelvic abscesses and

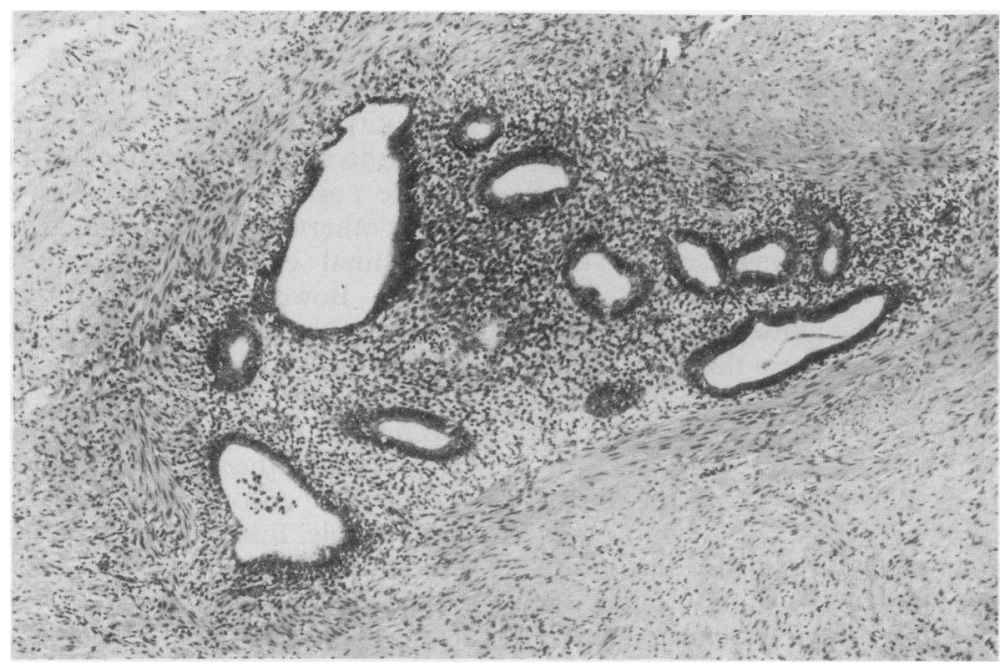

Fig. 2. Endometrial glands and stroma in the muscle of the bowel wall, the classical appearance of endometriosis $(\times 38)$. 


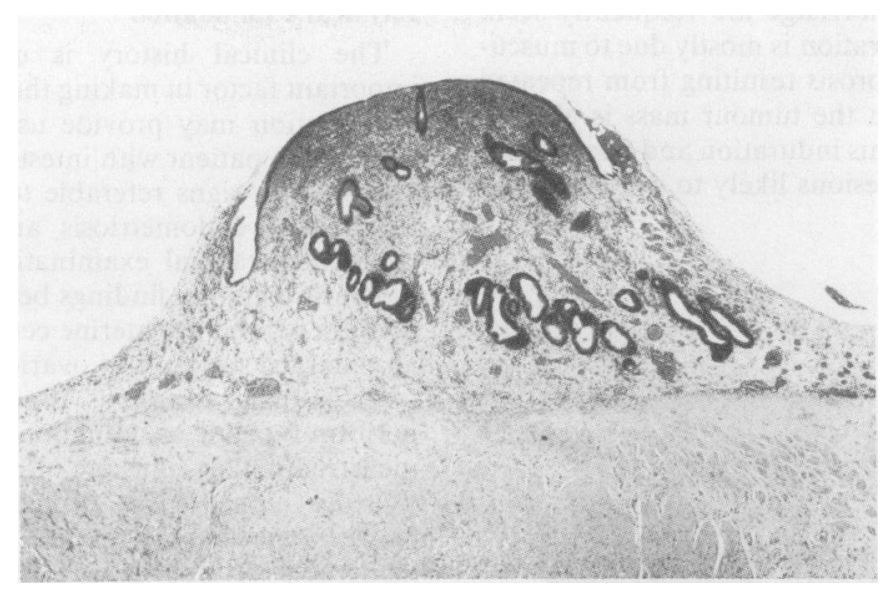

FIG. 3. A serosal deposit of endometriosis ( $\times 96)$.

haematomas. Goldman and Minkin (1980) were unable to make an unequivocal diagnosis in any of their cases, finding ultrasound extremely accurate however, in determining the size, shape and location of endometriosis in known cases.

Laparoscopic diagnosis is now commonplace and the characteristic appearances have been summarized by Kistner et al., (1977) and Cohen $(1979,1980)$. The additional benefits of the laparoscope are that biopsies may be taken to obtain histological confirmation (Cohen, 1979, 1980), superficial implants may be cauterized and minor adhesions divided. Histological diagnosis in certain cases may be essential as coexistent malignant disease can occur (Macafee and Greer, 1960).

\section{Management}

Pre-operative diagnosis of intestinal endometriosis carries considerable practical importance as most authors advocate that medical management should be attempted for mild to moderate cases (Kistner $\boldsymbol{e t}$ al., 1977; Cohen, 1979, 1980). Oestrogens, progestogens, androgens, oestrogen/progestogen combination and danazol in varying regimes have all been prescribed. Mainly due to their side effects, oestrogens and androgens have.fallen from favour. Progestogens enjoyed widespread popularity in the early 1970s but their usage has declined, the commonest current treatment being the use of the oestrogen/progestogen combinations in the form of the birthcontrol pill, or danazol.

It is in severe endometriosis or following the failure of medical management, that surgery plays an important role in the gynaecological management of the disease (Kistner et al., 1977; Williams, 1975; Andrews, 1980). Williams (1975) divides surgery for endometriosis into 'conservative' and 'definitive' cat- $\vec{A}$ egories. Conservative surgery is defined as any procedure performed for endometriosis in which the child-bearing capacity of the patient is maintained. Definitive surgery refers to total hysterectomy and bilateral oophorectomy with excision or destruction $\stackrel{5}{\stackrel{5}{4}}$ of all known areas of endometriosis. When a pres $\overrightarrow{\overrightarrow{0}}$ operative diagnosis of intestinal endometriosis has been made it is important to consider definitive. surgery at the time of operation in order to avoid a further laparotomy at a later date.

If classical symptoms are not elicited in the history, often due to the fact that previous gynaecological symptoms or treatment have been overlooked by the $\AA$ patient, the surgeon may be faced at operation with a $\overrightarrow{\vec{B}}$ lesion of the bowel closely mimicking carcinoma, and $\exists$ an absence of other signs such as peritoneal or ovarian endometriotic deposits. Previous gynaecological surgery should alert the surgeon to the possibility of endometriosis even if a typical history is 3 not forthcoming, but in all cases a frozen section may provide positive confirmation of endometriosis and should otherwise exclude malignancy.

Intestinal endometriosis may require extensive surgery. Bowel preparation is advisable, and the Pfannenstiel approach is contraindicated (Kistner et al., 1977).

In the case of complete intestinal obstruction, resection is the operation of choice (Macafee and Greer, 1960), other deposits being completely excised N or cauterized and definitive surgery performed when- N ever possible. Bilateral oophorectomy alone may be a 0 suitable alternative to bowel resection in the older

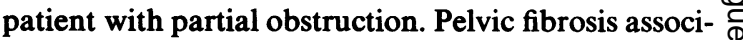
ated with ovarian and pouch of Douglas endome- $\stackrel{9}{\rightarrow}$ triosis may considerably distort the course of the ureter which should be identified and mobilized 
before bowel resection (Kistner et al., 1977). Williams (1975) has suggested that the peritoneal cavity and laparotomy incision be irrigated with isotonic saline to minimize the chance of implantation of endometrial fragments.

The postoperative hormonal treatment of endometriosis, if definitive surgery has not been performed, involves inducing a pseudopregnancy with oestrogens and progestogens, or a pseudomenopause with danazol, the subject being reviewed by Kistner et al., (1977) Cohen, (1979, 1980) and Andrews (1980).

\section{Conclusion}

Endometriosis is a disease of increasing incidence. Pre-operative diagnosis helps to avoid unnecessarily radical bowel resection and inadequate gynaecological surgery. Intestinal involvement may occur without a classical gynaecological history or presentation. It is therefore necessary to consider the possibility of intestinal endometriosis in any post-menarchal female patient with abdominal symptoms.

\section{References}

ANDREWS, W.C. (1980) Medical versus surgical treatment of endometriosis. Clinical Obstetrics and Gynecology, 23, 917.

BURNS, F.J. (1967) Endometriosis of the intestine. Diseases of Colon and Rectum, 10, 344.
COHEN, M.R. (1979) Laparoscopy and the management of endometriosis. Journal of Reproductive Medicine, 23, 81 .

COHEN, M.R. (1980) Laparoscopic diagnosis and pseudomenopause treatment of endometriosis with danazol. Clinical Obstetrics and Gynecology, 23, 901.

Goldman, S.M. \& Minkin, S.I. (1980) Diagnosing endometriosis with ultrasound: accuracy and specificity. Journal of Reproductive Medicine, 25, 178.

GRAY, L.A. (1973) Endometriosis of the bowel: role of bowel resection, superficial excision and oophorectomy in treatment. Annals of Surgery, 177, 580.

Kistner, R.W., Siegler, A.M. \& Behman, S.J. (1977) Suggested classification for endometriosis: relationship to infertility. Fertility and Sterility, 28, 1008.

MACAFEE, C.H. \& GREeR, H.L. (1960) Intestinal endometriosis: A report of 29 cases and a survey of the literature. Journal of Obstetrics and Gynaecology of the British Commonwealth, 67, 539.

MORSON, B.C. \& DAwSON, I.M.P. (1979) Gastrointestinal Pathology. 2nd edn. Blackwell Scientific Publications, Oxford.

RANNEY, B. (1975) The prevention, inhibition, palliation and treatment of endometriosis. American Journal of Obstetrics and Gynecology, 123, 778.

RANNEY, B. (1980) Endometriosis: Pathogenesis, symptoms and findings. Clinical Obstetrics and Gynecology, 23, 865.

SANDLER, M.A. \& KARO, J.J. (1978) The spectrum of ultrasonic findings in endometriosis. Radiology, 127, 229.

WILliams, T.J. (1975) The role of surgery in the management of endometriosis. Mayo Clinic Proceedings, 50, 198.

Williams, T.J. \& PratT, J.H. (1977) Endometriosis in 1000 consecutive coeliotomies; Incidence and management. American Journal of Obstetrics and Gynecology, 129, 245.

(Accepted 15 February 1984) 